\title{
Tunable water-based metasurface for anomalous wave reflection
}

\author{
Jacobsen, Rasmus Elkjær; Nielsen, Jonas Ø.; Lavrinenko, Andrei V; Arslanagi, Samel
}

Published in:

Journal of Physics D: Applied Physics

Link to article, DOI:

$10.1088 / 1361-6463 / a b b 2 b b$

Publication date:

2020

Document Version

Peer reviewed version

Link back to DTU Orbit

Citation (APA):

Jacobsen, R. E., Nielsen, J. Ø., Lavrinenko, A. V., \& Arslanagi, S. (2020). Tunable water-based metasurface for anomalous wave reflection. Journal of Physics D: Applied Physics, 53(50), [505104 ].

https://doi.org/10.1088/1361-6463/abb2bb

\section{General rights}

Copyright and moral rights for the publications made accessible in the public portal are retained by the authors and/or other copyright owners and it is a condition of accessing publications that users recognise and abide by the legal requirements associated with these rights.

- Users may download and print one copy of any publication from the public portal for the purpose of private study or research.

- You may not further distribute the material or use it for any profit-making activity or commercial gain

- You may freely distribute the URL identifying the publication in the public portal

If you believe that this document breaches copyright please contact us providing details, and we will remove access to the work immediately and investigate your claim. 


\title{
Tunable Water-Based Metasurface for Anomalous Wave Reflection
}

\author{
Rasmus E. Jacobsen ${ }^{1}$, J. $\emptyset$. Nielsen ${ }^{2}$, Andrei V. Lavrinenko ${ }^{1}$, and Samel Arslanagić ${ }^{2}$ \\ ${ }^{1}$ Department of Photonics Engineering, Technical University of Denmark, 2800 Kgs. Lyngby, Denmark \\ ${ }^{2}$ Department of Electrical Engineering, Technical University of Denmark, 2800 Kgs. Lyngby, Denmark \\ E-mail: rajac@fotonik.dtu.dk
}

Received xxxxxx

Accepted for publication $\mathrm{xxxxxx}$

Published xxxxxx

\begin{abstract}
Metasurfaces are artificial structures which enable a full control of electromagnetic waves and they continue to be of great scientific importance. In particular, they can tailor their reflection and transmission properties by using electrically small metallic/dielectric scatterers with imprinted spatial phase variation. In the present work, we design and characterize a waterbased metasurface with a tunable reflection at $1 \mathrm{GHz}$. The metasurface comprises a collection of water cylinders and we demonstrate its ability to reflect a normally incident wave at an angle of $51.3^{\circ}$ and with a total reflection efficiency of $33 \%$. A prototype was fabricated and is shown to exhibit an excellent agreement with the simulation results. The proposed water-based metasurface may serve as a cheap and bio-friendly tunable alternative for a variety of microwave communication systems.
\end{abstract}

Keywords: water, liquid, metasurface, reflectarray, bio-friendly, reconfigurable

\section{Introduction}

Various devices have been demonstrated since the introduction of metasurfaces (MSs) [1-3]. Especially alldielectric versions of the latter have paved the way for efficient wave tailoring of visible and infrared light. However, a limiting factor at such high frequencies is the availability of high-permittivity materials required to realize these structures. This is not a problem at radio and microwave frequencies, where many high-permittivity ceramics are commercially available [4]. Recently, another high-permittivity candidate was proposed, namely water, as an abundant, cheap and environmentally friendly alternative for microwave metamaterials and MSs as well as dielectric resonator antennas (DRAs), absorbers and heating systems, to mention a few [5-16]. With its frequency- and temperature-dependent high permittivity as well as its liquid form, water introduces numerous tuning parameters enabling easy tuning of the devices. The losses in water can, however, be an issue at some frequencies consequently reducing its potential in a variety of potential applications. Yet in a time with increasing demand for environmentally friendly products, water holds the potential to be a fundamental element in ecological compatible microwave MSs and even bulk metamaterials.

Two special types of wave transformers used in antenna applications are the reflectarray and transmitarray, which are non-uniform surface structures enabling the control of the reflected and transmitted waves, respectively. Their working principle relies on discrete spatial phase jumps across their surfaces effectively altering the angle of the reflected/ transmitted wave. Many designs, both metallic and dielectric, have been demonstrated recently [1-2], [17-20], even though the first devices are dated long before [21-22], and have their historical roots in the antenna theory.

In this work, we design and characterize a MS reflectarray with subwavelength displaced array elements composed of simple cylindrical cavities filled with distilled water. Through proper disposition of the water content in each cavity, the reflected wave direction can be tailored. Presently, a MS with a period (termed a supercell) of eight unit-cells (each consisting of a water cavity in a low-permittivity host) is investigated for the possibility of reflecting a normally incident wave away from the normal and in a direction governed by the generalized Snell's law of reflection. The required phase shift across the MS is obtained as a result of a horizontal magnetic dipole resonance induced in the water 
cylinders. The operating frequency is $1 \mathrm{GHz}$, and the unit-cell size and the thickness are, respectively, 6 and 20 times smaller than the free-space wavelength. The MS was characterized numerically in COMSOL Multiphysics, and a protype was built for experimental demonstration. The plane wave spectrum of the MS is calculated to evaluate the efficiency, bandwidth and polarization sensitivity. The proposed MS may serve as a simple and versatile as well as extremely cheap and environmentally friendly component (e.g. a reflectarray) for microwave communication systems.

The paper is organized as follows. Section II presents the configuration of the proposed MS. It also includes a discussion on its design as well as on the obtained numerical and experimental results. Section III includes a summary and conclusions of this work. Throughout the work, the timefactor $\exp (j \omega t)$, where $\omega$ is the angular frequency, and $t$ is the time, is assumed and suppressed.

\section{Configuration and results}

One period of the MS is sketched in figure 1. It consists of $N$ cylindrical distilled water elements in a rectangular cuboid Rohacell $51 \mathrm{RF}$ host medium with a conducting ground plane on the backside to cancel the transmission. A rectangular coordinate system $(x, y, z)$ is introduced as shown in figure 1 . The water cylinders have equal radii $(r=20 \mathrm{~mm})$ and different heights $\left(h_{n}\right.$ with $\left.n=1,2, \ldots, N\right)$, and are spaced by a distance $a$ along the $x$-axis. The supercell is periodic in the $x y$-plane with the lattice constants $N a$ and $a$. The MS is designed to reflect a linearly $x$-polarized plane wave at normal incidence. The angle of the reflected plane wave is denoted $\theta_{\mathrm{r}}$. All results are for $20^{\circ} \mathrm{C}$ water temperature.

The simulations were performed in COMSOL Multiphysics [23] with a simple model consisting of a rectangular periodic 1-port system with the supercell incorporated; the ground plane was emulated as a Perfect Electric Conductor (PEC) effectively cancelling the transmission. A Perfect Matched Layers (PML) is inserted on the backside of the port and the incident electric field magnitude is set to $E_{0}=1 \mathrm{~V} / \mathrm{m}$. We used a model for the permittivity of water [24], and at $1 \mathrm{GHz}$ the relative permittivity is $79.9-j 4.5$. For the Rohacell $51 \mathrm{HF}$ material, we used the relative permittivity of 1.075 as reported in our previous work [10].

\subsection{The metasurface design}

Generalized Snell's law of reflection states that

$$
\sin \theta_{\mathrm{r}}-\sin \theta_{\mathrm{i}}=\Delta \phi / k_{0} a,
$$

where $\theta_{\mathrm{i}}$ and $\theta_{\mathrm{r}}$ are the angles of the incident and reflected waves, respectively, $\Delta \phi=360^{\circ} / N$ - is the phase jump between the cylinders in the supercell, $k_{0}=2 \pi / \lambda_{0}$ - is the free-space wavenumber and $\lambda_{0}-$ is the free-space wavelength.

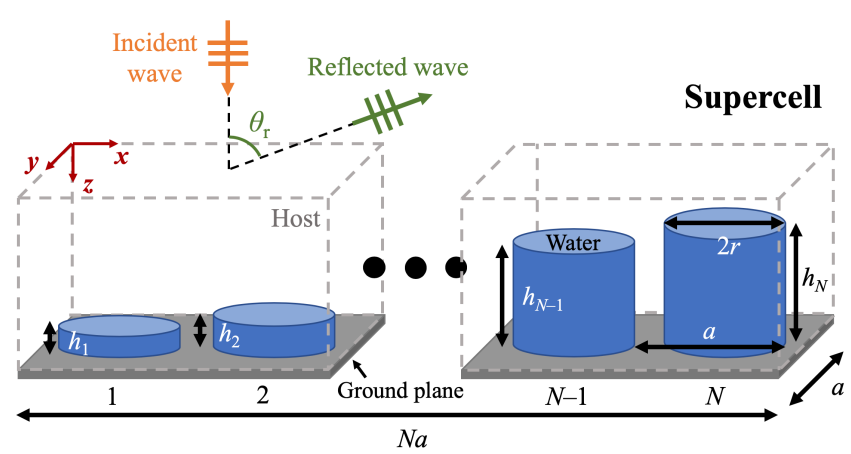

Figure 1. Sketch of one supercell of the MS.

The law shows that by introducing a phase-shift of $360^{\circ}$ across the supercell, the angle of reflection can be tailored at will. As a consequence of the periodicity of the supercell, several orders of diffractions/reflections can exist as long as their reflection angles $\theta_{\mathrm{r}, m}<90^{\circ}$ with integer $m$ being the order. This is the same principle behind diffraction gratings [25]. Presently, we focus on the first order reflection $(m=1)$. We introduce discrete phase jumps by varying the height of the water cylinders across the supercell. First, the cylinders providing the required phase shift to the reflected wave were determined by considering the unit cell of a square lattice $(N$ $=1$ ) of identical cylinders with lattice constant $a=48 \mathrm{~mm}(\approx$ $\left.\lambda_{0} / 6\right)$. A plane wave at normal incidence is investigated, and due the symmetry and periodicity of the MS, only normally reflected plane waves are generated. The height of the cylinders was then swept, and the resulting squared magnitude and phase of the reflection coefficient $\left(\mathrm{S}_{11}\right)$ are shown in figure 2(a) for heights ranging from 6 to $20 \mathrm{~mm}$. Approximately $330^{\circ}$ phase shift is achieved albeit with a reduced reflection magnitude. This is coming from a magnetic dipole induced in the water cylinders, which is on resonance for $h=10.7 \mathrm{~mm}$, where the minimum in the reflection coefficient, i.e., maximum absorption, is. The total magnetic field magnitude (colors) and the normalized electric field (arrows) in the $x z-$ plane of a resonant water cylinder with $h=10.7 \mathrm{~mm}$ are shown in figure 2(b) exhibiting the characteristics of a magnetic dipole resonance: The electric field is mirrored by the PEC ground plane and circulates near the periphery inside the water cylinder generating an enhanced magnetic field.

We also studied variation of the radius (result is not included), and the result is similar to that in figure 2(a). However, we found the MS with cylinders of varying heights and fixed radius to be much easier to fabricate.

With the frequency and $a$ being fixed, the supercell is constrained to $N>6$ cylinders for $\theta_{\mathrm{r}}<90^{\circ}$. Presently, a MS with a super cell of $N=8$ cylinders is investigated, which has the expected first order reflection angle of $\theta_{\mathrm{r}}=51.3^{\circ}$. Higher order reflections do not exist as their reflection angles would be larger than $90^{\circ}$. Cylinders with phase jumps of $\Delta \phi=45^{\circ}$ were selected for the MS and the height for each cylinder is listed in table 1. 


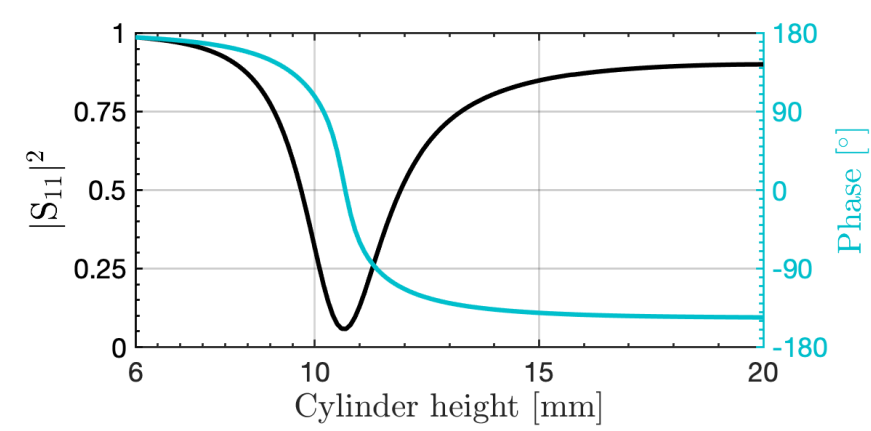

(a)

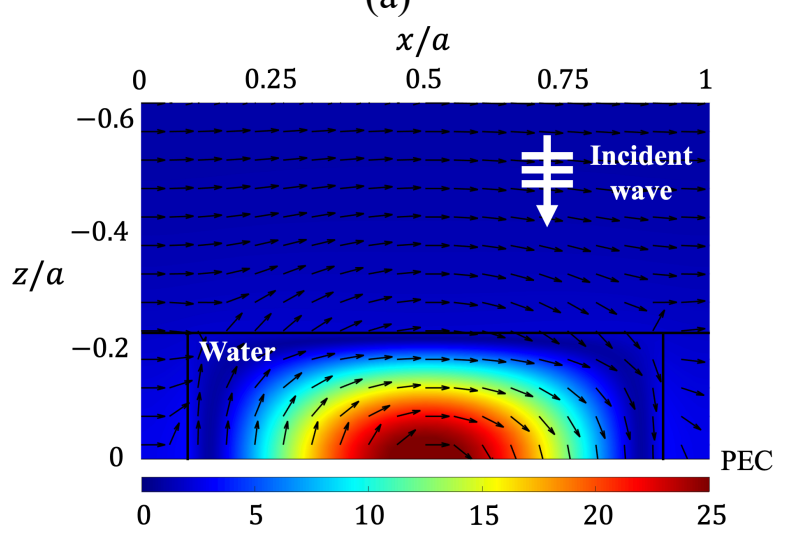

(b)

Figure 2. Numerical investigation of the square lattice of identical cylinders $(N=1)$. (a) The squared magnitude and relative phase of the reflection coefficient $\left(\mathrm{S}_{11}\right)$ as functions of the cylinder height. (b) The total magnetic field magnitude (colors) in the $x z$-plane of the resonant cylinder with $r=20$ $\mathrm{mm}$ and $h=10.7 \mathrm{~mm}$. The arrows in (c) show the direction of the electric field.

\subsection{Performance of metasurface}

The performance of the infinite MS is evaluated by its plane wave spectrum. A simple numerical procedure was developed for the purpose of computing the discrete Fourier transform of the complex reflected field, $\boldsymbol{E}^{\mathrm{r}}(x)$, along a line parallel to the $x$-axis. The line was truncated at $100 \mathrm{Na}$ to obtain a sufficient resolution. The transformed field represents the $k_{\mathrm{x}}$-component of each plane wave travelling in different directions $k_{\mathrm{x}}=$ $k_{0} \sin \theta_{\mathrm{r}}$. Due to the infinite extent of the MS in the $(x, y)$ direction, the reflected power flowing away from the array must be $-z$-directed i.e., only the $z$-component of the reflected time-average power flow density, $S_{z}^{\mathrm{r}}(\theta)=$ $S_{R}^{\mathrm{r}}\left(\theta_{\mathrm{r}}\right) \cos \theta_{\mathrm{r}}$, contributes to the far-field [17]. The reflection efficiency is then calculated as

$$
\eta_{\text {eff }}=S_{\mathrm{z}}^{\mathrm{r}}\left(\theta_{\mathrm{r}}\right) / S_{0}
$$

where $S_{0}=\left|E_{0}\right|^{2} / 2 \eta_{0}$ is the time-average power flow density of the incident wave with $\eta_{0}$ being the intrinsic impedance of free space. Similarly, the reflected time-average power flow
Table 1. Heights of the water cylinders for the MS.

\begin{tabular}{ccccccccc}
\hline $\begin{array}{c}\text { Cylinder } \\
\text { no. }\end{array}$ & $\mathbf{1}$ & $\mathbf{2}$ & $\mathbf{3}$ & $\mathbf{4}$ & $\mathbf{5}$ & $\mathbf{6}$ & $\mathbf{7}$ & $\mathbf{8}$ \\
\hline $\begin{array}{c}\text { Height } \\
{[\mathrm{mm}]}\end{array}$ & 6.0 & 9.6 & 10.2 & 10.5 & 10.7 & 10.9 & 11.5 & 14.7 \\
\hline \hline
\end{tabular}

density is calculated as $S_{R}^{\mathrm{r}}\left(\theta_{\mathrm{r}}\right)=\left|\widetilde{\boldsymbol{E}}^{\mathrm{r}}\left(\theta_{\mathrm{r}}\right)\right|^{2} / 2 \eta_{0}$ with $\widetilde{\boldsymbol{E}}^{\mathrm{r}}\left(\theta_{\mathrm{r}}\right)$ being the Fourier transform of $\boldsymbol{E}^{\mathrm{r}}(x)$.

The $\eta_{\text {eff }}$ efficiency as a function of $\theta_{\mathrm{r}}$ is shown in figure 3 (a) for both $x$ - and $y$-polarized plane wave incidences. The theoretical $\theta_{\mathrm{r}}=51.3^{\circ}$ is shown by the red circles and the MS has a dominating reflection of $\eta_{\text {eff }}=33 \%\left(\eta_{\text {eff }}=27.5 \%\right)$ at that exact angle for the $x$-polarized ( $y$-polarized) incidence. All other reflections are much smaller; there is a reflection at $\theta_{\mathrm{r}}=0^{\circ}\left(\theta_{\mathrm{r}}=-51.3^{\circ}\right)$ with $\eta_{\text {eff }}=4 \%\left(\eta_{\mathrm{eff}}=0.1 \%\right)$ for the $x$ polarized incidence. Summing all reflected plane waves gives the total reflected power of $37 \%$. The remaining power must be absorbed by water, and this is confirmed by integrating the power loss density over the volume of the supercell, where the absorption is found to be $63 \%$. Another performance metric used for reflective surfaces is the relative reflection efficiency, which is the reflected power in the desired direction divided with the total reflected power. For the present MS, this is 89 $\%$ for the $x$-polarized incidence.

Although the MS is anisotropic, there is only small difference between the $x$ - and $y$-polarized incidences. This is due to the isotropic water elements, which makes the MS only slightly polarization sensitive. The cross-polarization of the MS was also investigated and was near non-existing due to the structure symmetry and the subwavelength spacing of the cylinders.

The instantaneous reflected electric field in the $(x, z)$-plane is shown in figure 3(b), with the arrows showing the direction of the normalized time-average reflected Poynting vector, plainly displaying a pronounced reflected plane wave travelling at an oblique angle away from the MS. The reflected wave is not uniform, which indicates that some cylinders absorb more power than others as was the case with the squared array in figure 2(a).

The bandwidth of the oblique reflection peak $\left(\theta_{\mathrm{r}}>0^{\circ}\right)$ is investigated in figure 4 with $\eta_{\text {eff }}$ and $\theta_{\mathrm{r}}$ as functions of frequency. The theoretical $\theta_{\mathrm{r}}$, calculated with equation (1), as well as $\eta_{\text {eff }}$ for the normal reflection $\left(\theta_{\mathrm{r}}=0^{\circ}\right)$, is also included in the figure. From equation (1), it is obvious that $\theta_{\mathrm{r}}$ will change with frequency as can be seen in figure 4 , and the simulated $\theta_{\mathrm{r}}$ exhibits excellent agreement with the theory. For frequencies away from $1 \mathrm{GHz}$, the normal reflection increases dramatically, and the oblique reflection is reduced. The halfpower bandwidth of the MS is $14 \%(140 \mathrm{MHz})$ with a $7^{\circ}$ variation of $\theta_{\mathrm{r}}$.

A prototype of the MS was fabricated at the local workshop. The prototype consisted of four supercells each with eight cylinders milled in blocks of Rohacell $51 \mathrm{HF}$ that were put 


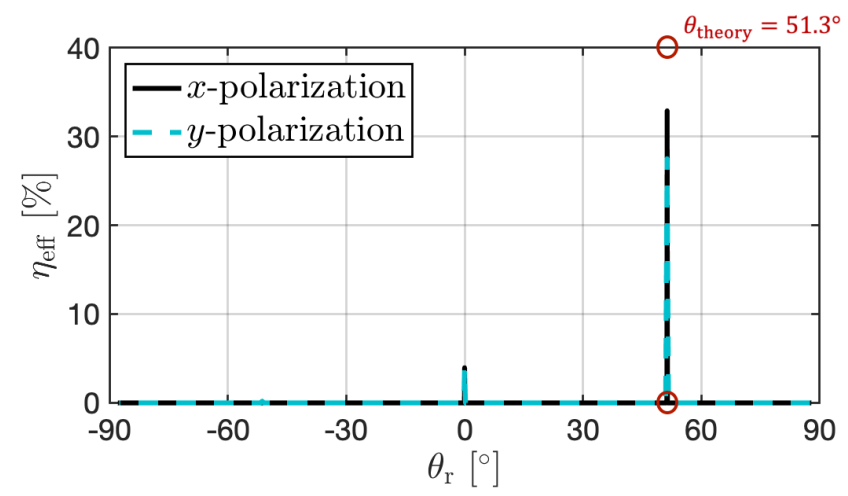

(a)

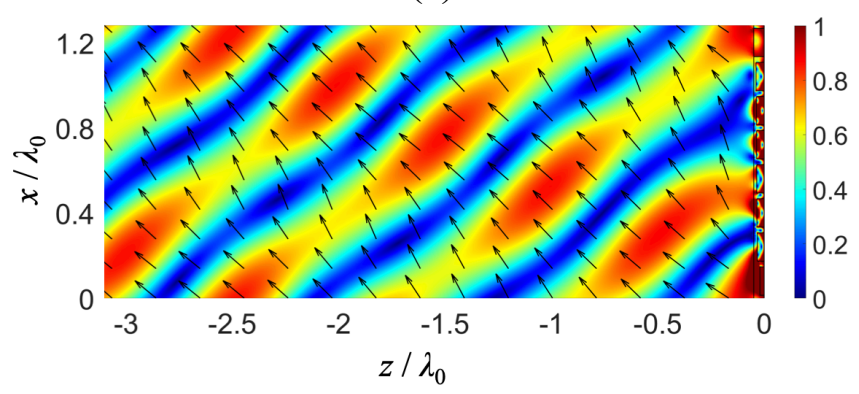

(b)

Figure 3. Results for the MS at $1 \mathrm{GHz}$. (a) Reflection efficiency as a function of the reflection angle for $x$ - and $y$ polarized plane wave incidences. The red circles show the theoretical reflection angle $\theta_{\text {theory }}=51.3^{\circ}$. (b) Magnitude of the instanteneous reflected field (colors) in the $x z$-plane of one supercell. The arrows show the direction of the normalized time-average reflected Poynting vector.

together. An aluminum tape was attached on the backside of the blocks to realize the ground plane as well as for sealing of the water inside the cylinders. For each cylinder height listed in table 1, we calculated the volume $\left(V_{n}=\pi r^{2} h_{n}\right)$ and then distributed distilled water by using a syringe. A photograph of the fabricated MS is shown in figure 5(a), and a sketch of the measurement setup established at the measurement facility is shown in figure 5(b). The setup consisted of two ETSLindgren 3164-03 horn antennas mounted on a metal beam hoisted 2.64 meter above the floor with two tripods. The antennas constituted a two-port system with one antenna $\left(\mathrm{P}_{1}\right)$ pointing directly at the ground whereas the position and angle of the other antenna $\left(\mathrm{P}_{2}\right)$ were adjusted for various angular receptions. The MS was put on the ground below $\mathrm{P}_{1}$, and the measured $S_{11}$ and $S_{21}$ as functions of the angle is shown in figure 5(c). The power measured with the MS was normalized with the power measured without it, and for comparison the normalized electric far-field result from the simulation of the truncated MS is included in figure 5(c). It should be noted that in the simulation model, the size of the ground plane is much smaller than in the experimental setup. Furthermore, the experiment was not conducted in radio anechoic conditions. Still we observe fine agreement between the measurement and simulation. At an angle of $52^{\circ}$, an approximately $6 \mathrm{~dB}$

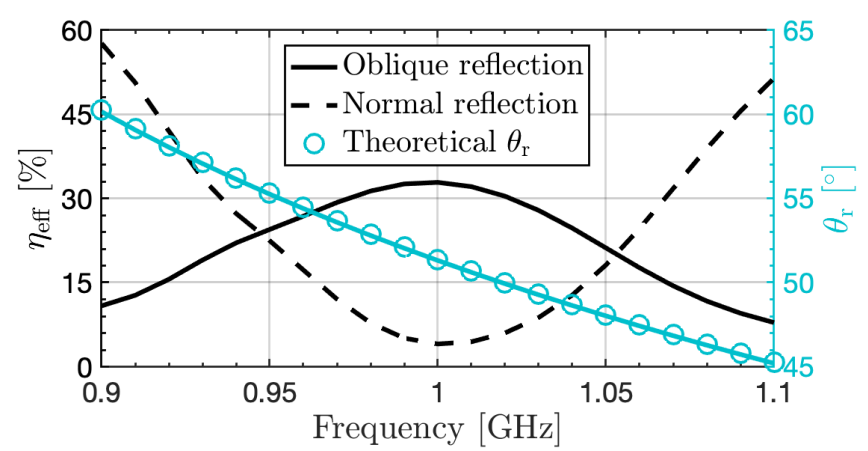

Figure 4. Reflection efficiency and angle as functions of frequency. The solid line shows the results for the oblique reflection peak, whereas the dashed line shows the result for the normal reflection $\left(\theta_{\mathrm{r}}=0^{\circ}\right)$. The circles show the theoretical $\theta_{\mathrm{r}}$ calculated with equation (1).

enhancement was measured in the experiment confirming the numerical results. Due to the small size of the MS, there is only a small reduction in the measured normal reflection of $-0.13 \mathrm{~dB}$.

The MS can easily be reconfigured for other $\theta_{\mathrm{r}}$ with just a simple re-distribution of water in the cylindrical cavities. Altering the size of the supercell to $N=7$, while maintaining the $360^{\circ}$ phase distribution across the supercell, predicts a $\theta_{\mathrm{r}}$ $=63^{\circ}$. Similarly, $N=9$ and $N=10$ predict the first (second) order $\theta_{\mathrm{r}}=44^{\circ}\left(88^{\circ}\right)$ and $\theta_{\mathrm{r}}=39^{\circ}\left(78^{\circ}\right)$, respectively. As $N$ increases, the $\theta_{\mathrm{r}}$ approaches $0^{\circ}$. Similarly, completely removing the water from the cavities will cancel the oblique reflection leaving only the normal reflection. In addition, the MS should potentially be reconfigurable for other types of wave transformations such as focusing [1-3], however this is not investigated in this work. We envision a piston system by which the height of the water cylinders can be controlled. Such a MS could be used as a tunable component in a base station or cheap amateur communication system for wave front control as well as higher directivity.

The present MS is designed for a $20^{\circ} \mathrm{C}$ water temperature, however, it will still function at $1 \mathrm{GHz}$ for temperatures few degrees below and above without the need for changing the water content in the cavities. As the temperature increases, the permittivity of water decreases consequently changing the response of the MS. With temperature changes, the operating frequency and reflection angle will change, e.g. for higher temperatures, the operating frequency is blue-shifted and the reflection angle is reduced. Alternatively, adding more water causes a red-shift, and in this way, the MS can be easily tuned for different water temperatures and/or frequencies. Additionally, $\eta_{\text {eff }}$ changes as well, due to the lower (higher) losses in water for higher (lower) temperatures. This was studied numerically: by increasing the water temperature in the MS to $50^{\circ} \mathrm{C}$, while adjusting the water content to maintain the phase distribution as well as the operating frequency at 1 $\mathrm{GHz}$; it resulted in a considerable increase in $\eta_{\text {eff }}$ to $61 \%$. 


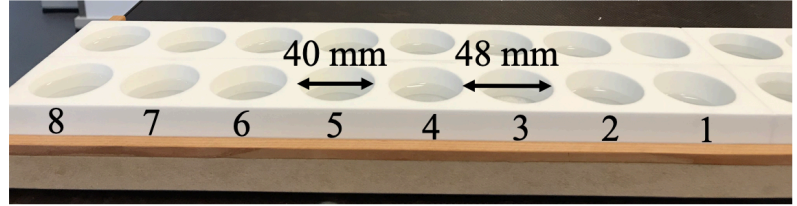

(a)

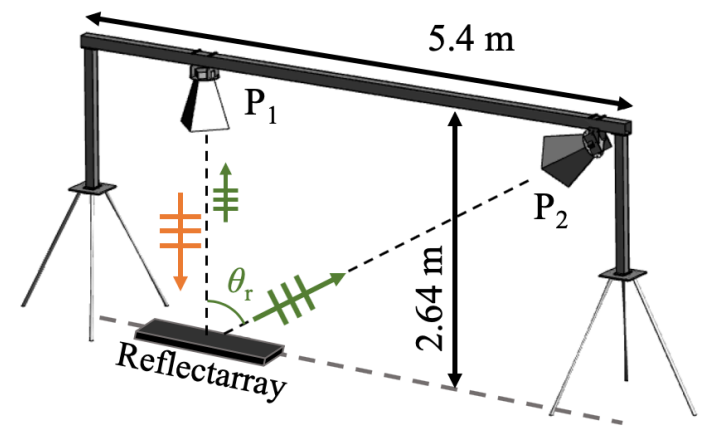

(b)

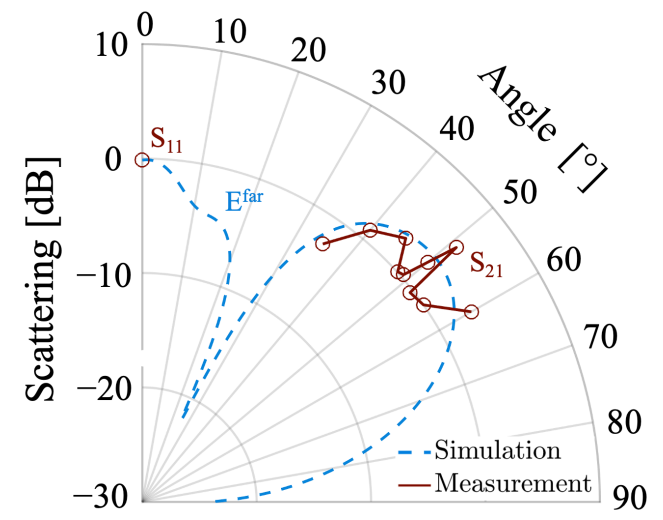

(c)

Figure 5. (a) Photograph of the fabricated MS, (b) sketch of the experimental setup and (c) measured normalized scattering shown in the logarithmic scale together with the normalized far-field simulation of the truncated MS.

Similar observations were made for a water-based dielectric resonator antenna [14].

\section{Summary and conclusions}

A MS consisting of simple cylindrical water-filled cavities in a Rohacell $51 \mathrm{HF}$ host was designed for $1 \mathrm{GHz}$ operation. Inside the water cylinders, magnetic dipoles are induced by the incident wave, bringing a complete change to the reflection. The MS converts $33 \%$ of the power of a normally incident plane wave into a reflected plane wave in the desired direction with $51.3^{\circ}$. This is achieved by distributing water across rows of cylinders such that a linear $360^{\circ}$ phase evolution is introduced to the reflected wave for each set of eight cylinders. The normally reflected power is minimized to $4 \%$, and $89 \%$ of reflected power is channeled into the desired direction. The polarization sensitivity of the MS is very low with only $5.5 \%$ difference in the reflected power between the $x$ - and $y$-polarizations. The half-power bandwidth is estimated to be $14 \%(140 \mathrm{MHz})$ with a $7^{\circ}$ variation of the reflection angle. A prototype of the MS was fabricated and characterized experimentally demonstrating the enhanced reflection in the desired direction.

The reduced efficiency of the MS is due to the strong resonance and losses in water. A simple way to improve the efficiency is to go to lower frequencies where the losses in water are significantly reduced. Similarly, increasing the water temperature will also reduce the losses in water, however, this would require the addition of a heating system. Alternatively, some of the water can be substituted with a different material like e.g. metal or low-loss dielectric.

The proposed MS cannot compete with existing microwave technology in terms of efficiency. Yet it is simple and versatile as well as extremely cheap and bio-friendly and may therefore serve as an alternative component for microwave communication systems. In addition, the Rohacell material used for the prototype is not essential for the functionality and can be replaced with a cheaper and more bio-friendly alternative. This mindset can be expanded to the complete design, such that all materials are ecological compatible that can be recycled or directly decomposed in nature.

By this work, we bring to public extremely simple realization of water-based MS, which can provide the same nomenclature of properties as 'solid' MS. Meanwhile, tunable properties are simply granted in our approach by easy reshaping, changing the volume or heating/cooling of water. Real-time tuning (modulation) can be done by applying ultrasound or acoustic waves with specific water containers. This subject is not so trivial for speculations, so extensive analysis is needed to prepare more or less sounding conclusion on possibilities of real-timing tuning at the level sufficient for microwave applications. It will be the topic of following studies.

\section{Acknowledgements}

The authors would like to thank the workshop at the Electromagnetic Systems group of the Technical University of Denmark for fabrication of the MS.

\section{References}

[1] N. Yu, F. Capasso. "Flat optics with designer metasurfaces," Nat. Mat., 13, 139-50 (2014).

[2] S. Jahani, and Z. Jacob, "All-dielectric metamaterials," Nat. Nanotechnol., 11, 23-36 (2016).

[3] H. T. Chen, A. J. Taylor, and N. Yu, "A review of metasurfaces: physics and applications,” Rep. Prog. Phys., 79, 076401 (2016).

[4] MARUWA, Ceramics products. Accessed on: December 6, 2019. [Online] Available: https://www.maruwag.com/e/products/ceramic/.

[5] M. A. Gorlach, M. Song, A. P. Slobozhanyuk, A. A. Bogdanov, and P. Belov, "Topological transition in coated wire medium," Phys. Status Solidi RRL, 10, 12, 900-904 (2016). 
[6] A. Andryieuski, S. M. Kuznetsova, S. V. Zhukovsky, Y. S. Kivshar, and A. V. Lavrinenko, "Water: promising opportunities for tunable all- dielectric electromagnetic metamaterials," Sci. Rep., 5, 13535 (2015).

[7] M. Odit, P. Kapitanova, A. Andryieuski, P. Belov, and A. V. Lavrinenko, "Experimental demonstration of water based tunable metasurface," Appl. Phys. Lett., 109, 011901 (2016).

[8] X. Cai et al., "Water based fluidic radio frequency metamaterials," J. Appl. Phys., 122, 184101 (2017).

[9] X. Yang et al., "Reconfigurable all-dielectric metasurface based on tunable chemical systems in aqueous solution," Sci. Rep., 7, 3190 (2017).

[10] R. E. Jacobsen, A. V. Lavrinenko, and S. Arslanagić, "Water-based metasurfaces for effective switching of microwaves," IEEE Antennas Wirel. Propag. Lett., 17, 4, 571574 (2018).

[11] Y. J. Yoo et al., "Metamaterial absorber for electromagnetic waves in periodic water droplets," Sci. Rep., 5, 14018 (2015).

[12] Y. Pang et al., "Thermally tunable water-substrate broadband metamaterial absorbers," Appl. Phys. Lett., 110, 104103 (2017).

[13] Y. Pang et al., "Water-based metamaterial absorbers for optical transparency and broadband microwave absorption," J. Appl. Phys., 123, 155106 (2018).

[14] R. E. Jacobsen, A. V. Lavrinenko, and S. Arslanagić, "Electrically small water-based hemispherical dielectric resonator antenna," Appl. Sci., 9, 4848 (2019).

[15] E. Motovilova, and S. Y. Huang, "A review on reconfigurable liquid dielectric antennas," Materials, 13, 8, $1863(2020)$.

[16] R. E. Jacobsen, S. Arslanagić, and A. V. Lavrinenko, "Continuous heating microwave system based on Mie resonances," in AMPERE 2019 Book of Abstracts, 160, 2019.

[17] V. S. Asadchy et al., "Perfect control of reflection and refraction using spatially dispersive metasurfaces," Phys. Rev. B, 94, 075142 (2016).

[18] A. Díaz-Rubio, V. S. Asadchy, A. Elsakka, and S. A. Tretyakov, "From the generalized reflection law to the realization of perfect anomalous reflectors," Sci. Adv., 3, 8, e1602714 (2017).

[19] R. Mao et al., "Tunable metasurface with controllable polarizations and reflection/transmission properties," J. Phys. D: Appl. Phys., 53, 15, 155102 (2020).

[20] L. Zhang et al., "Transmission-reflection-integrated multifunctional coding metasurface for full-space controls of electromagnetic waves," J Adv. Funct. Mater., 28, 33, 1802205 (2018).

[21] D. G. Berry, R. G. Malech, and W. A. Kennedy, "The reflectarray antenna," IEEE Trans. Antennas Propag., 11, 6, 645-651 (1963).

[22] D. M. Pozar, S. D. Targonski, and H. D. Syrigos, "Design of millimeter wave microstrip reflectarray," IEEE Trans. Antennas Propag., 45, 2, 287-296 (1997).

[23] COMSOL Multiphysics 5.3. 2017. [Online]. Available: https://www.comsol.com/.

[24] W. Ellison, "Permittivity of pure water, at standard atmospheric pressure, over the frequency range 0-25 thz and the temperature range 0-100 c.," J. Phys. Chem. Ref. Data, 36, 1-18 (2007).

[25] S. Larouche, and D. R. Smith, "Reconciliation of generalized refraction with diffraction theory," Opt. Lett., 37, 12, 2391-3 (2012). 